\title{
Multifractal analysis and multiagent simulation for market crash prediction
}

\author{
V. Romanov, V. Slepov, M. Badrina \& A. Federyakov \\ Plekhanov Russian Academy of Economics, Russia
}

\begin{abstract}
In this paper the results of multifractal analysis by means of partitions and scaling function calculation are described, as well as wavelet analysis, which were applied to USA 1987 October Black Monday DJ data. For the partition calculation and Legendre transform a special program was elaborated. As our aim is predicting crash situations, we are trying to find out the best indicator that uses multifractal analysis and wavelet analysis methodology. With this aim in mind we have tested different methods of preprocessing the original time series to discover the best indicator. The wavelet analysis data were calculated on a 256 day moving window. The changes in the multifractal analysis features were studied while approaching crisis point and after the crisis. From the multiagent market model we can observe the crisis evolution and the dynamic of changing parameters such as share prices, trading volumes, price increments and statistical distribution dependent on traders' strategies.
\end{abstract}

Keywords: stock market, market dynamics, multiagent simulation, wavelet analysis.

\section{Introduction}

The prediction of tough crucial changes in the finance market is very difficult because of the non-linear structure of the processes. It doesn't allow us to effectively use common statistical methods. Hurst exponent values estimation can be used for time series properties determination and for choosing the proper method of data processing. Unfortunately in the case of the multifractal time series, the Hurst exponent value is not constant and at different time scales takes different values, generating a spectrum of values.

Multifractal analyses potentially give us the possibility of predicting sharp changing market states. In this paper we are making an attempt to apply 
mathematical theories and methods that are known from the literature [1-7] for detection of approaching crises situations at the stock market. Results of the experiment on processing real and artificial data by this special program, realizing Gibb's partition method and fractal dimension spectrum estimation, are presented in this article. It was also shown that wavelet analysis with Daubichies-12 expansion gives another possibility for approaching crises detecting.

\section{Methodology}

\subsection{Scaling function estimation}

It is known that fixed time scale is inappropriate for analysis of complex dynamic systems such as the stock market. For estimating the fractal dimension spectrum the partitions method may be used. Let us have time series $\left\{x_{t}\right\}$; $t \in[0, T]$. On the interval $[0, T]$, we compute the logarithmic price increment:

$$
Z=\left\{z_{t}\right\}, z_{t}=\ln x_{t+1}-\ln x_{t}, t=0, \ldots, T
$$

We divide interval $[0, T]$ on $\mathrm{N}$ subintervals, where $N$ integer, $l \leq N \leq N_{\max }$, so that each segment will contain $\operatorname{int}\left(\frac{T}{N}\right)=A$ values of time series $Z_{t}$. With such a partition the total amount of processed readings will be $A N$. Let us denote the segment index $K$, where $1 \leq K \leq N$. For each segment let us denote the current number of readings $l_{K}$, running in the range $1 \leq l_{K} \leq A$. The correspondence between indexes $l_{K}$ and $t$ is:

$$
t=(K-1) A+l_{K}
$$

In the literature [1-7] an opinion is expressed that the spectrum of the local Hölder exponent is widening at the moments, preceding the crash and it's plot widths jump sharply after the crash. So the aim of our research is to discover observable distinctions between time series fractal parameters before and after the crash point. These differences may be used later as early detectors and warnings of stock market crashes. As we are looking for the best possible indicators, we will test several methods of preprocessing the original time series. We consider the following variants:

-the original time series itself $Z_{0}=\left\{z_{t}\right\}$;

-the preprocessed time series (accumulated sum at each interval) $Z_{1}=\left\{\hat{Z}_{K}\right\}, K=1,2 \ldots N$, where

$$
\hat{Z}_{K}=\frac{1}{A} \sum_{l K=1}^{A} Z_{\kappa_{l_{K}}}
$$

-the preprocessed time series (subtract the mean and divide by the mean square deviation)

$$
Z_{2}=\left\{\frac{Z_{(K-1) A+l_{K}}-\hat{Z}_{K}}{S_{K}}\right\}
$$




$$
S_{K}=\sqrt{\frac{1}{A} \sum_{l_{K}=1}^{A}\left(Z_{K, l_{K}}-\hat{Z}_{K}\right)^{2}}
$$

-the preprocessed time series (subtract mean)

$$
Z_{3}=\left\{Z_{(K-1) A+l_{K}}-\hat{Z}_{K}\right\}
$$

The partition gives us the possibility of representing the time series in different time scales, so we take one reading from each interval and in such a way we compress the scale.

Then we compute one dimensional array for every fixed $\mathrm{N}$ and for different values q, $Q_{\min } \leq q \leq Q_{\max }$ - the scaling function for all four variants:

$$
\begin{gathered}
\hat{\tau}_{N}^{0}(q)=\frac{\log \pi^{(0)}{ }_{N}(Z, q)+\log A-\log N}{-\log A} \\
\hat{\tau}_{N}^{1}(q)=\frac{\log \pi^{(1)}{ }_{N}(Z, q)+\log A-\log N}{-\log A} \\
\hat{\tau}_{N}^{2}(q)=\frac{\log \pi^{(2)}{ }_{N}(Z, q)+\log A-\log N}{-\log A} \\
\hat{\tau}_{N}^{3}(q)=\frac{\log \pi^{(3)}(Z, q)+\log A-\log N}{-\log A}
\end{gathered}
$$

For all four variants we estimate the local Lipschitz-Hölder exponents describing the singularity strength as a two dimensional array for different values of $q$ :

$$
\alpha_{i}=\frac{d \hat{\tau^{i}}}{d q}=\left(\hat{\tau}^{i}(q)-\hat{\tau}^{i}(q-1)\right) / \Delta q=\Delta \hat{\tau}^{i}(q) / \Delta q, i=1,2,3,4 .
$$

Carrying out the Legendre transform of scaling function $\tau(q)$ we find multifractal singularity spectrum $f(\alpha)$ as the Hausdorff dimensions of the fractal subset with Lipshitz-Hölder local exponent $\alpha$ :

$$
f_{i}(\alpha)=\arg \min _{q}\left[q \alpha-\hat{\tau}_{i}(q)\right] \approx\left(\left[q \Delta \hat{\tau}^{i}(q) / \Delta q-\hat{\tau}^{i}(q), i=1,2,3,4\right.\right.
$$

For determining the position of $\tau(q)$ plot we use properties $\tau(0)=-1$ and $\tau\left(\frac{1}{H}\right)=0$. From another side, $\tau(q)$ is a monotonically increasing function. We can regard the scaling function as the slope of the partition function in bilogarithmic coordinates. In expression (12) the Legendre transform expresses reciprocal correspondence between statistic moments and the singularity exponent. The width of $\alpha$ spectrum may be estimated as different between the maximum and minimum values of $\alpha$ :

$$
\Delta \alpha=\alpha_{\max }-\alpha_{\min }
$$


Spectrum analysis of the time series interval near the crash events may detect the presence of market forecasting signals. Despite the partition method making it possible to explicate global stable relations, as we stated, it not sensitive enough to fast local changes. This method also demands significant value of calculations and elaboration of corresponding software. Wavelet analysis is to some extent free from these drawbacks.

\subsubsection{Wavelet analysis of multifractal time series}

Wavelet analysis doesn't use the assumption of stationary and ergodic properties of the time series, and like the partition method wavelet analysis allows us to analyze the time series behaviour at different time scales. Due to the compactness of the carrier, wavelet analysis can detect short living events at short time segments as a trend at long intervals.

Wavelet is a function with zero mean and finite energy. Unlike the Fourier analysis the wavelet analysis method operates locally in time and doesn't need cycle stationary components. The wavelet transform may be presented as an integral expression:

$$
W(\tau, \alpha)=\int_{-\infty}^{+\infty} x(t) \psi_{\tau, \alpha}(t) d t
$$

where $\psi_{\tau, \alpha}(t)$ is the function with zero mean centred around zero with time scale $\alpha$ and time horizon $\tau$. A family of wavelet vectors is created from the mother function by displacement and scaling:

$$
\psi_{\tau, \alpha}(\mathrm{t})=\frac{1}{\sqrt{\alpha}} \psi\left(\frac{t-\tau}{\alpha}\right)
$$

The scaling functions re the set of function $\varphi_{k}$ composing the span of subspace $V_{0}$ of space $L^{2}(R)$. So any function $f(t) \in V_{0}$. $V_{0}$ may be represented as a linear combination of scaling functions. Any discrete signal $f(t) \in L^{2}(R)$ may be represented in wavelet function terms:

$$
\begin{gathered}
\mathrm{f}(\mathrm{t})=\sum_{k=-\infty}^{\infty} \alpha_{j_{0}} \phi_{j_{0}, k}(t)+\sum_{j \geq j_{0}} \sum_{k=-\infty}^{\infty} \beta_{j, k} \psi_{j, k}(t) \\
\alpha_{j_{0}, k}=\int f(t) \phi_{j_{0}, k}(t) d t \\
\beta_{j, k}=\int f(t) \psi_{j, k}(t) d t
\end{gathered}
$$

where $j_{o}$ is a constant, representing the highest level of resolution for which the most acute details are extracted.

The decomposition process of discrete values segments into averaged or approximating values and detail values for different scales are named as multiscale or multiresolution analysis. Multiresolution analysis is invariant to integer value translations and distortions, which are proportional to the power of two. 
We have performed some experiments on processing real time series using multifractal analyses as wavelet analyses. The method of multifractal analyses, described above, has also been applied for the October 1987 USA financial crises, using Dow Jones index (Fig. 1).

Fig. 2 shows fractal dimension spectrum F2 $(\alpha)$, which have been calculated according to (12).

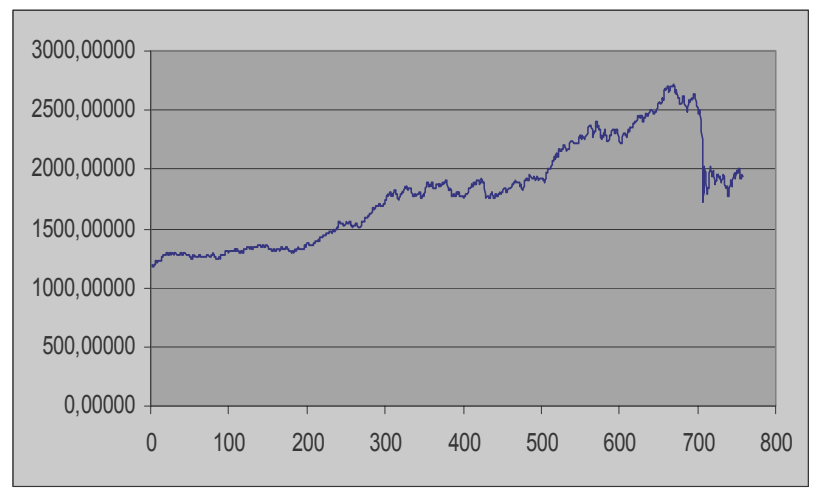

Figure 1: Dow Jones industrial average data for period 01.02.198531.12.87. Axis X contains serial numbers of readings.

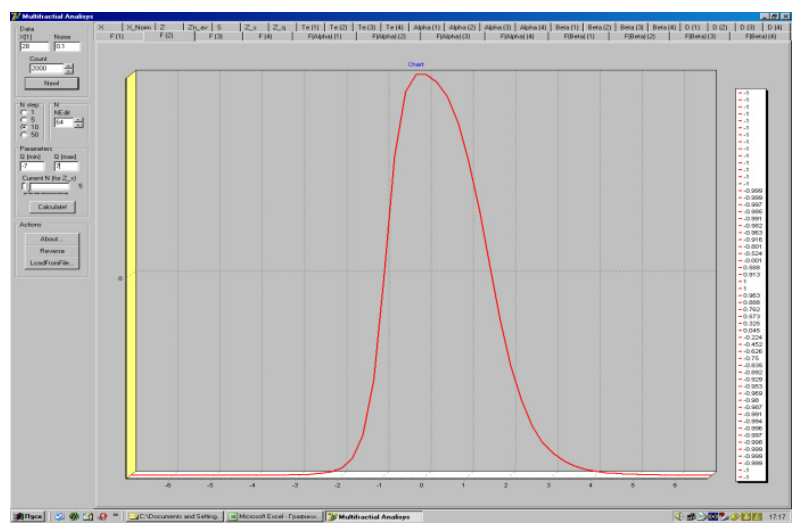

Figure 2: $\quad$ Fractal dimension spectrum F2 $(\alpha)$ for DJ industrial average series for period 10.10.85-19.10.87.

Fig. 3 and Fig. 4 show the plots of the multifractal spectrum width changing before and after crisis. The plots of different colours correspond to different levels of intersection of the fractal dimension spectrum graph and the lines of constant values $0,0.6,0.8$.

From these plots we can see that there are peaks, both for the F1( $\alpha)$ and F2 $(\alpha)$ versions of the multifractal spectrum preceding to the crises point and 
corresponding to dates 08.10 .87 for $F 1(\alpha)$ and 07.10 .87 for $F 2$, which permit us to assert that there are some kinds of predictive signals at least 8 days before crises. However, we continued the search of crisis indicators the using wavelet analysis method. In our research we choose Daubichies wavelets. Daubichies wavelets fit very well for coding of sequences, having intervals of slow changing or relatively constant values. From another side, Daubichies wavelets have some number of zero moments, which allow one to discriminate thin details.

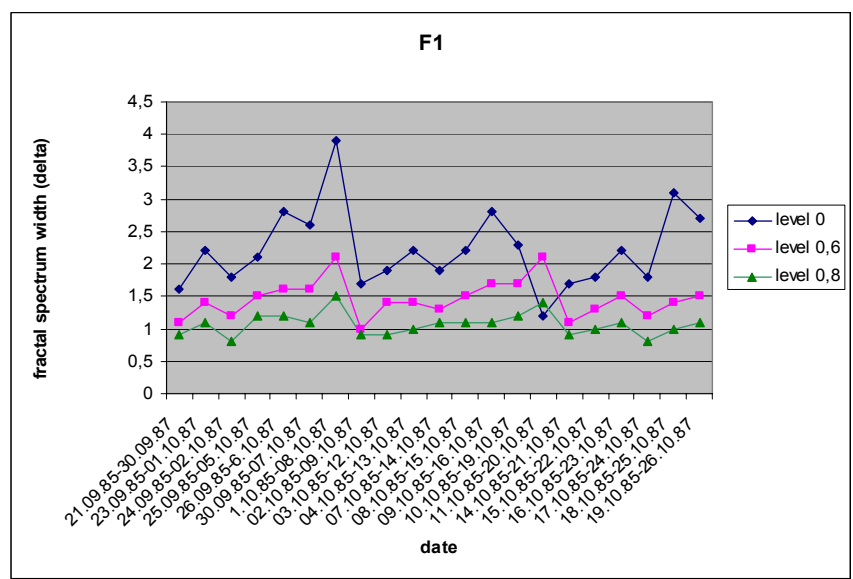

Figure 3: $\quad$ Fractal dimension spectrum width F1 $(\alpha)$ changing before and after crises.

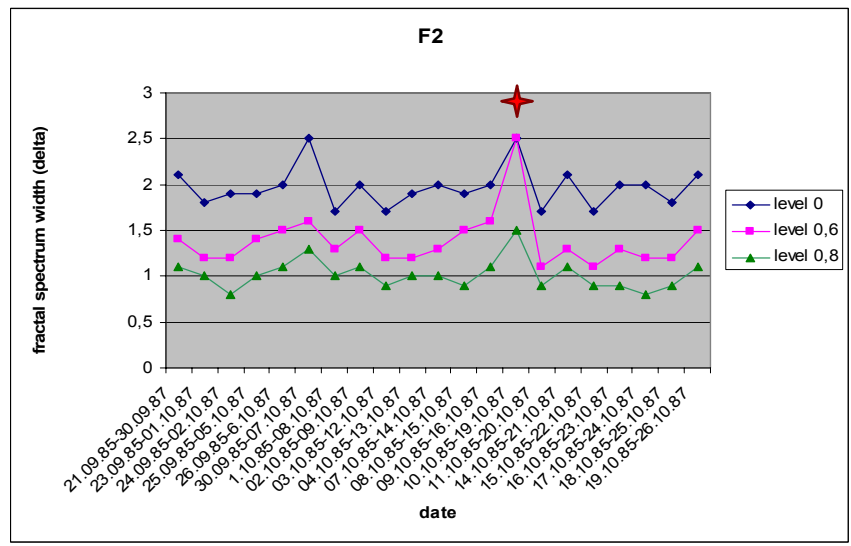

Figure 4: $\quad$ Fractal dimension spectrum width F2 $(\alpha)$ changing before and after crises. 
The aim of the experiments was to extract a signal by means of time series processing, which could predict the following sharp changes in time series. We use Daubichies-12 expansion approximation and detail coefficients for 5 levels. We have processed 21 intervals each containing 256 readings from 02.09 .86 29.10.87. For each interval we find the expansion coefficient at all 5 levels. Then we take the differences of maximum values from adjusting the intervals. The plots of maximum values and their differences are presented in Fig. 5 and Fig. 6.

The curve in Fig. 5 has a maximum at point 12.10 .87 . At the same point in Fig. 6 the curve is intersecting axis X. So we can make a conclusion that in both cases we obtain some indicators (maxima points) of forthcoming crisis, but these indicators are relatively weak. Obviously, this information can be used to detect a coming crash.

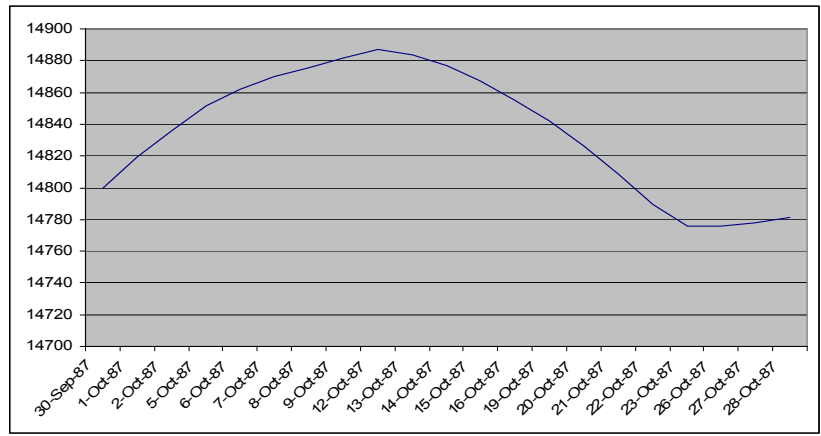

Figure 5: The plot of changing maximum values detail coefficients Daubichies -12 expansion.

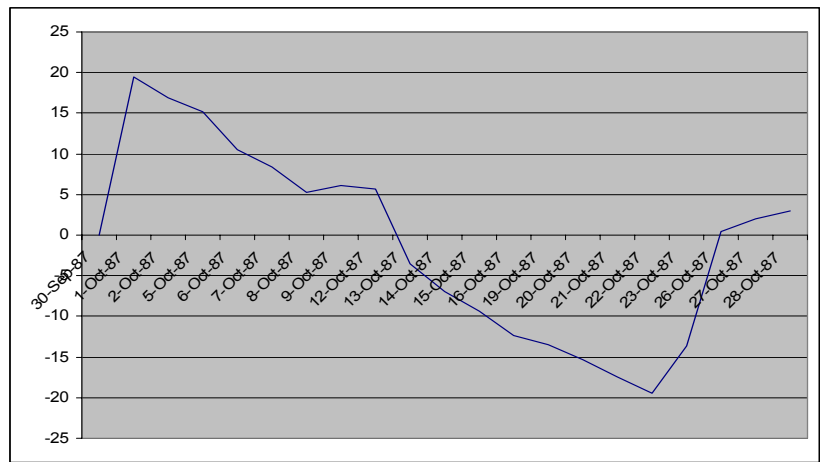

Figure 6: The plot of maximum differences.

In order to study how the crisis evolves in time we have made a financial market multiagent model, which was partly published in [8]. The choice of initial parameters of a virtual market makes sure that the virtual market is a reduced copy of an average real market. This in some way guarantees that the virtual 
market will behave in a similar way to the real market during the experiments. In this case, shown in Fig. 7, we used the following parameter values:

MARKET MAKER TRADER_COUNT $=2$;

RANDOM ${ }^{-}$TRADER COUNT $\overline{-}=0$;

FUNDAMENTAL_TRADER_COUNT $=500 ;$ BROKER_COUNT $=5$;

MARKET_COUNT $=1$;

COMPANY_COUNT $=10$;

COMPANY_MAX_ASSETS $=50000000 ; / / 50 \mathrm{kk}$

COMPANY_MIN_ASSETS $=1000000 ; / / 1 \mathrm{kk}$

MIN_BROKER_MARKET_ACCOUNT_MONEY = 100000; // 100k.

MAX_BROKER_MARKET_ACCOUNT_MONEY $=150000 ; / / 300 \mathrm{k}$.

BROKER_MONEY $=10000 ; / / 10 \mathrm{k}$.

The experiment is based on the "FundamentalTraders.fms" scenario. Its goal is to determine how fundamental traders' actions can influence share price changes.

The main goal of fundamental traders in the system is to make the share price follow some predefined "want-to-be" price. There are several events in this scenario: at first the company increases its assets, then it decreases them very quickly, and at the end of this 3-month experiment it again increases them. Traders' fundamental price is directly linked with companies' assets, so we can expect that the market price will be influenced by traders' expectations and somehow be linked to the fundamental 1 price too. In Fig. 7 we can observe how an artificial "crisis" is evolving in time: a period of significant price jumping is changed by comparatively modest deviations.

In Fig. 8 price distributions are presented. As we can see, share prices distribution is very close to distributions on the real markets. Only the average price distribution has a rather low peak value. Also the last transaction price has a very high peak. It may have been caused by high spread value or a very high

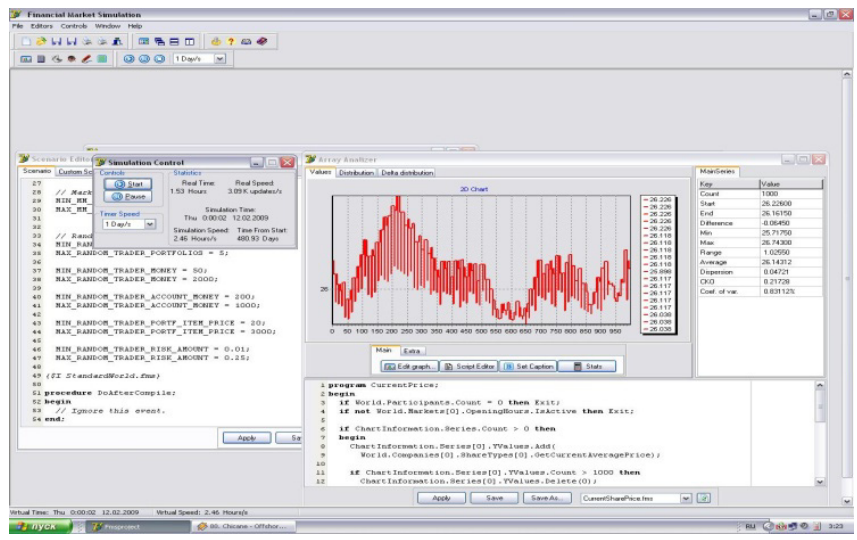

Figure 7: $\quad$ Stock market dynamic model. 


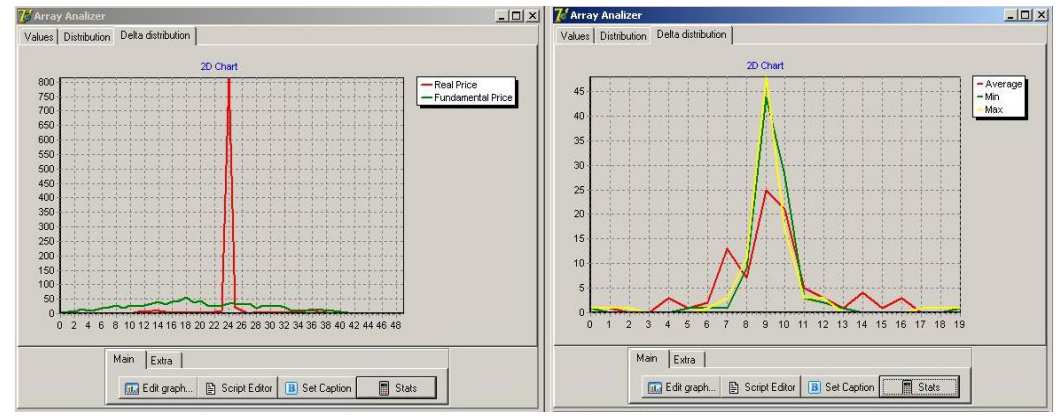

Figure 8: Distribution of share prices in the "FundamentalTraders.fms" scenario.

number of transactions (around 1000). It's a well known fact - the longer the initial array, the higher peak on its distribution. Almost flat distribution of fundamental price is an expected result, because it is generated from the company's assets, which change based on a simple algorithm that uses the standard Delphi random number generator.

\section{Conclusion}

In spite of positive research results in the detection of forthcoming crash situations by means of multifractal as wavelet analysis, there are some difficulties in getting appropriate patterns for adjusting the model's parameters. So it seems reasonable to use an agent based market simulation for generating artificial data, containing some patterns, which may be further applied for improving multifractal and wavelet models.

This approach can promote investigation in the domain of forecasting market time series by means of supplying necessary data for exploring forecasting methods market time series behaviour.

\section{References}

[1] Cornelis A. Los (EMEPS Associates, Inc.), Rossitsa M. Yalamova (University of Lethbridge), Multifractal spectral analysis of the 1987 stock market Crash. http://papers.ssrn.com/sol3/papers.cfm?abstract_id=588823

[2] Stefan Reimann, "An elementary model of price dynamics in a financial market Distribution, Multiscaling \& Entropy". http://ideas.repec.org/p/ zur/iewwpx/271.html

[3] Thomas Lux: "Detecting multi-fractal properties in asset returns: The failure of the 'scaling estimator"'. http://ideas.repec.org/p/zbw/cauewp/ 1124.html

[4] Seong-Min Yoon, Kyungsik Kim and J. S. Choi: "Multifractal Features in Ko-rean Financial Markets". http://cat.inist.fr/?aModele=afficheN\&cpsidt $=16772737$. 
22 Computational Finance and its Applications III

[5] Francois Schmitt, Shaun Lovejoy: "Multifractal fluctuations in finance". http://arxiv.org/abs/cond-mat/0102369

[6] Marco Corazza, Anastasios G. Malliaris: "MultiFractality in Foreign Currency Markets". http://sba.luc.edu/research/wpapers/990303.pdf

[7] I. A. Agaev, Yu. A. Kuperin: "Multifractal Analysis and Local Hoelder Expo-nents Approach to Detecting Stock Markets Crashes". http://arxiv.org/abs/cond-mat/0407603

[8] http://sourceforge.net/projects/fimas 